\title{
Patient satisfaction with the annual wellness visit
}

Maria Espiridion $^{1}$, Raghavendra Mulinti ${ }^{2}$, Suzanne E. Kemper ${ }^{3}$, Lynne J. Goebel ${ }^{1}$

\section{Author Affiliations:}

1. Marshall University, Huntington, West Virginia

2. West Virginia University, Morgantown, West Virginia

3. Charleston Area Medical Center, Charleston, West Virginia

The authors have no financial disclosures to declare and no conflicts of interest to report.

\section{Corresponding Author:}

Lynne J. Goebel

Marshall University

Huntington, West Virginia

Email: goebel@marshall.edu 


\section{Abstract}

Objectives - To determine patient satisfaction with the Annual Wellness Visit (AWV)

Design - Survey

Setting - Charleston Area Medical Center and Marshall Health

Participants - 66 volunteers, average age 74 years

Measurements - Patient satisfaction was assessed with a 13 item survey.

Results - Of 1,537 eligible patients, 211 (14\%) of these agreed to schedule their AWV. From 66 patients surveyed, $87 \%$ of patients said the visit "met expectations," "would recommend to friends," and "would do it again." Only $5(8 \%)$ were disappointed that new problems were not addressed and $2(3 \%)$ were dissatisfied that physical exams and blood tests were not included. Conclusion - Our hypothesis that patients would not be satisfied with their visit was not supported. A benefit of the visit was that patients planned on following through with new recommendations. Future research should address the impact of a recommendation from primary care providers on patient acceptance of the AWV.

\section{Keywords}

Annual Wellness Visits, Affordable Care Act, Patient Satisfaction

\section{Introduction}

In 2011, the Patient Protection and Affordable Care Act made the Annual Wellness Visit (AWV) available without co-pay for eligible Medicare beneficiaries. ${ }^{1,2}$ The AWV is conducted by a physician or another non-physician medical professional and aims to establish a health risk assessment by screening for problems with physical and cognitive function and delineating the risks to be addressed in the patient's prevention plan. ${ }^{3-5}$ Review of the literature reveals no published works regarding patient satisfaction with the AWV. Previous research revealed the efficacy of patient satisfaction surveys and the benefits they provide for identifying patients' needs, however they were not specific to the AWV. ${ }^{6-9}$ We hypothesized that patients may not be satisfied completely with the AWV possibly because it does not include a physical exam or address new complaints. We hoped to determine patient satisfaction with the AWV and identify areas for improvement.

\section{Methods}

IRB approval was obtained for this multi-site study involving Marshall Health and Charleston Area Medical Center (CAMC) with CAMC deferring to Marshall University IRB for approval.

Marshall Health information technology department compiled a list of internal medicine attending and resident physician patients with Medicare coverage who visited their primary care provider within the preceding twelve months and did not have the AWV in the last year.

We contacted 1,574 patients by telephone in July 2014 with 1,537 of these being eligible as they were age 65 and older and received care at the general internal medicine or Hanshaw geriatric clinics at Marshall Health and had not had an AWV in the past year. Patients were informed that the purpose of the call was to schedule the AWV. If the patient agreed to the visit, their 
information was given to front desk staff to schedule a 60-minute appointment with a physician assistant who conducted all AWVs at Marshall Health.

A CAMC geriatrician performed AWVs during his regularly scheduled office hours on patients new to him who had not had the wellness visit in the past year and had no other complaints. His visits lasted 40 to 60 minutes.

At the end of their AWV, providers handed patients a consent form explaining the purpose of the survey, along with the 13 item survey instrument. Patients recorded their gender, age, quality of overall health, if they were patients of Marshall Health or CAMC, and if this was their first wellness visit. Following these demographic questions were 11 other items regarding patient satisfaction with the AWV. We selected the survey questions after reading other patient satisfaction surveys ${ }^{10,11}$ and adding some items that were specific to the AWV. Each item was answered on a 5-point Likert scale ( $1=$ strongly agree, $5=$ strong disagree). We tested the survey for face validity with people who were not eligible for the AWV. We scanned surveys with an optical reader and entered survey data into an Excel spreadsheet.

\section{Data Analysis}

Data analysis was completed using SAS version 9.3 (SAS Institute, Inc, Cary, NC). Basic descriptive statistics using frequencies and proportions were used to analyze survey responses. Chi square analysis or Fisher's exact test, when appropriate, was used to compare responses between sub groups of survey participants. An alpha level of less than 0.05 was used to determine statistical significance.

In the data evaluation, strongly agree and agree answers were combined as were strongly disagree and disagree. Following the 11 items were two sections that allowed participants to comment on areas of the AWV that they particularly enjoyed and provide any suggestions for improvement. We grouped the responses into different categories based on the major theme of the response. The categories included comments on the provider conducting visit, information provided, and time spent with the healthcare provider.

\section{Results}

\section{Telephone invitation results}

We contacted 1,537 eligible Marshall Health patients by telephone, and 211 (14\%) of these agreed to schedule their AWV. Of the 211 respondents, $38 \%$ were male. The 1,326 who did not agree to schedule their AWVs offered many different spontaneous responses. Although 438 (33\%) of these patients offered no reason for declining, the remaining 888 participants stated that they: 1) found no reason to come in because they already had a primary care provider (509, $38 \%)$, 2) had too many physicians and other healthcare providers to see $(369,28 \%)$, or 3 ) were skeptical of and/or disliked the Affordable Care Act (10, 1\%).

Survey results 
66 surveys were distributed and all 66 returned following the AWV, 41 from Marshall Health and 25 from CAMC. The average age of the respondents was 74 years, and the majority of these patients were female $(45,68 \%)$. Almost three quarters of the participants reported this was their first AWV $(49,74 \%)$. More than one third $(26,39 \%)$ of patients rated their overall health as "very good" and almost half $(31,47 \%)$ of the patients rated their health as "fair." 
Table 1. Survey results ${ }^{\mathrm{a}}$

Survey Question

The Annual Wellness Visit was too long

The Annual Wellness Visit met my expectations

I would recommend this visit to

friends and family

I would make this visit again next

year

The information provided to me was not very useful

I am satisfied that a physical exam was not part of the visit

I am satisfied that blood tests were not part of the visit

I was disappointed that I did not get the chance to discuss other or new problems I was having at this visit

The healthcare provider found new information about the medications I was taking during this visit Frequency Missing = 1

The healthcare provider made new recommendations regarding preventive care during this visit

I am planning on making changes or otherwise following at least some of the suggestions made by the healthcare provider

\begin{tabular}{|c|c|c|}
\hline $\mathrm{N}(\%)$ Strongly Agree/Agree & $\begin{array}{c}\mathrm{N}(\%) \text { Neither } \\
\text { Agree or } \\
\text { disagree }\end{array}$ & $\begin{array}{c}\mathrm{N}(\%) \text { Disagree/Strongly } \\
\text { disagree }\end{array}$ \\
\hline $2(3 \%)$ & $24(36 \%)$ & $40(61 \%)$ \\
\hline $58(88 \%)$ & $7(11 \%)$ & $1(2 \%)$ \\
\hline $57(86 \%)$ & $6(9 \%)$ & $3(5 \%)$ \\
\hline $58(88 \%)$ & $7(11 \%)$ & $1(2 \%)$ \\
\hline $16(24 \%)$ & $8(12 \%)$ & $41(62 \%)$ \\
\hline $47(71 \%)$ & $17(26 \%)$ & $2(3 \%)$ \\
\hline $47(71 \%)$ & $17(26 \%)$ & $2(3 \%)$ \\
\hline $5(8 \%)$ & $17(26 \%)$ & $44(67 \%)$ \\
\hline $21(32 \%)$ & $18(27 \%)$ & $26(39 \%)$ \\
\hline $41(62 \%)$ & $17(26 \%)$ & $8(12 \%)$ \\
\hline $53(80 \%)$ & $9(14 \%)$ & $4(6 \%)$ \\
\hline
\end{tabular}

a Total $\mathrm{N}=66$ 
The majority of patients reported the AWV met their expectations $(58,88 \%)$, they would recommend this visit to friends and family $(57,86 \%)$, and they would make the visit again next year $(58,88 \%)$ (Table 1$) .41(62 \%)$ patients reported receiving new recommendations during their visit, $21(32 \%)$ patients reported the provider discovered new information about their medications, and $53(80 \%)$ patients plan on making changes or otherwise following at least some of the suggestions made by the healthcare provider.

Only 2 patients (3\%) reported that the AWV was too long and 5 patients $(8 \%)$ stated they were disappointed that they did not get the chance to discuss other or new problems during the visit. Just 2 patients $(3 \%)$ were dissatisfied that blood tests were not part of the visit and that a physical exam was not part of the visit. Despite the positive feedback received, almost one quarter (16, $24 \%$ ) of the patients stated that the information they received from the healthcare provider during the AWV "was not useful."

Respondents aged 65-74 ( $\mathrm{n}=35)$ were more likely to agree that they would recommend the visit to friends and family than those 75 years and older $(n=24)(p=0.008)$. The younger group was also more likely to agree that they were satisfied that a physical exam was not part of the visit $(p=0.04)$. There were no significant differences in answer to the questions according to selfreported health status.

Four patients gave spontaneous responses to the question "How could your visit be made better?" Those responses were to have a list of tests included in the AWV, to do bloodwork before the visit, to have a physical exam, and to discuss diet. In response to the question that asked patients "What did you like best about your visit?" 11 made positive comments about the provider, 8 liked the information they received, and 2 were happy with the time spent with the provider.

\section{Discussion}

Patients who participated in the AWV had a positive experience and were satisfied with the visit and their healthcare providers. One possible reason for the satisfaction may be due to the one hour allotted visit time patients had with a healthcare provider, which allowed time for clarification of recommendations and development of a preventive healthcare plan in a detail that they might not typically receive during their usual primary care visit due to time constraints. Importantly, patients reported they liked the information received including new recommendations made during the visit, and if they follow through with the recommendations as planned this would be an important benefit.

The intended AWV model was such that acute problems would not be addressed at the visit. In order to comply with visit time constraints, providers in this study chose not to address new problems during the AWV, although we realize this is possible and could be billed using an appropriate code. A small subset of patients returning the survey did report their disappointment with not having new problems addressed and not having a physical exam or blood work during their visit. Although consistent with our original hypothesis, this was a much smaller problem than we anticipated. 
It was surprising that so few patients agreed to have the AWV. There are some barriers to the AWV that will need to be overcome in order to improve acceptance of the visit. Some elderly see the AWV as a burden because, as in the Marshall Health practice, it required another visit and patients questioned the need to see another provider. Patients may defer to their primary care providers to see if they really need this visit. Our study showed possible benefits of this visit for patients for whom new preventive care recommendations were made. If physicians realize that this visit is helpful, they may be more likely to recommend the visit. Future study could look at the impact of the primary care providers' recommendation during an office visit on patient acceptance of the AWV. Our study used a telephone call invitation, but the investigator who called was not familiar to the patient and this may have had a negative impact on uptake of the visit. If the insurance companies are insistent that patients have this visit and patients are not convinced they need the visit, then a reward such as a gas card to cover the cost of transportation to the visit might be offered.

There was one question on the survey asking participants to agree or disagree with the statement that "the information provided during the AWV was not useful." Despite having a largely positive response from the participants on other questions, almost a quarter of patients recorded that the information they received was not useful. We feel that it is possible these patients may not have answered this question accurately due to the negative wording in the question which required participants to read more closely.

Another limitation of this study was the small survey population. One reason for the small number of patients was the fact that many people did not agree to schedule the visit. The patients who did agree to the visit may be different from those who refused and this may have biased the survey results as these patients wanted this visit. Another barrier is finding provider time for this lengthy visit. The CAMC site used a geriatrician to perform the AWV during follow up visits where the patient did not have any acute complaints. In geriatric practice, this doesn't happen very often. The Marshall Health site used a physician assistant whose schedule was easier to adapt to this type of visit. Additionally, since the physician assistant in this study is not the primary care provider, patients may be less likely to bring up new problems so the preventive nature of the visit could be preserved. Future study could involve a component of provider satisfaction with the visit to see if this is an issue.

Finally, it is important to discuss the limitation of having different healthcare providers at different sites. It is unclear whether patients had concerns regarding the type of healthcare provider who conducted their visit or how this affected their satisfaction with the visit. This could be addressed in future studies with larger numbers of patients and providers of different types. We did not compare results between the two sites due to small numbers of patients involved.

\section{Conclusion}

Our hypothesis that patients would not be satisfied with their AWV was not supported. Patients reported a positive experience with their wellness visits and planned to continue them. The AWV may be beneficial as new recommendations were made and most patients planned on following through with these recommendations. Some patients, however, see the visit as a burden and 
practices that use providers other than the primary care provider to perform the AWV may see resistance in scheduling the visit. 


\section{References}

1. Preventive visit \& yearly wellness exams [Internet]. Baltimore, MD: Centers for Medicare \& Medicaid Services. [cited 2014 July 15]. Available at: http://www.medicare.gov/coverage/preventive-visit-andyearly-wellness-exams.html.

2. Affordable Care Act [Internet]. Washington, D.C.: U.S. Department of Health \& Human Services [cited 2014 July 15]. Available at: http://www.hhs.gov/healthcare/rights/law/index.html.

3. The Myers Group [Internet]. [cited 2014 July 15]. Available at: http://www.themyersgroup.net/clinician.asp.

4. National Research Corporation [Internet]. [cited 2014 July 15]. Available at: http://www.nationalresearch.com.

5. Zgierska A, Rabago D, Miller MM. Impact of patient satisfaction ratings on physicians and clinical care. Patient Prefer Adherence 2014 Apr 3;8:437-446.

6. White B. Measuring Patient Satisfaction: How to Do It and Why to Bother. Fam Pract Manag. 1999 Jan;6(1):40-44.

7. Hojat M, Louis DZ, Maxwell K, Markham FW, Wender RC, Gonnella JS. A Brief Instrument to Measure Patients' Overall Satisfaction With Primary Care Physicians. Fam Med. 2011 Jun;43(6):412-417.

8. Schoenfelder T, Klewer J, Kugler J. Determinants of patient satisfaction: a study among 39 hospitals in an in-patient setting in Germany. Int J Qual Health Care. 2011 Oct;23(5):503-509.

9. Bowling A, Rowe G, McKee M. Patients' experiences of their healthcare in relation to their expectations and satisfaction: a population survey. J R Soc Med. 2013 Apr;106:143-149.

10. CSQ Scales [Internet]. [cited 2014 July 15]. Available at: http://www.csqscales.com/csq-8.htm.

11. Marshall GN, Hays RD. The Patient Satisfaction Questionnaire Short Form (PSQ-18). Santa Monica, CA:Rand Corporation; 1994:1-31. 